\title{
An Assessment of WiMax Security
}

\author{
Sanjay P. Ahuja, Nicole Collier \\ School of Computing, University of North Florida, Jacksonville, USA \\ E-mail: \{sahuja, nicole.collier\}@unf.edu \\ Received February 23, 2010; revised April 20, 2010; accepted April 29, 2010
}

\begin{abstract}
For a broadband wireless standard such as WiMax, security is important and must be addressed. This is to ensure wide acceptance both from the perspective of the end users and the service providers. In order to compete with existing broadband cable or DSL services, the WiMax network must offer comparable security. We discuss the WiMax security mechanisms for authentication, encryption, and availability. We also discuss potential threats to WiMax security. This paper will also discuss how and why these threats play an important role in the adaptability of WiMax.
\end{abstract}

Keywords: WiMax, WiMax Security, Service Provider

\section{Introduction}

With the introduction of Wireless LANs in the 90s network security became a very important subject of discussion among major corporations, service providers, and end users. Security in wireless networks is the maintaining of confidentiality, authentication, non-repudiation, and integrity control [1]. To keep these four areas protected from malicious attacks, certain protocols were put into place. These protocols are constantly being tested and improved as necessary to keep corporations safe from outside, and sometimes inside users. These protocols are also being used by home users as more wireless networks are being brought into the homes of end users.

Worldwide Interoperability for Microwave Access (WiMax) is described as "a standards based technology enabling the delivery of last mile wireless broadband access as an alternative to cable and DSL" [2]. WiMax provides many services using point to point and point to multipoint applications. These applications are cost effective and cover a much larger area than WiFi (IEEE 802.11). WiMax uses a base station to transmit to Customer Premise Equipment (CPE). Using a base station allows WiMax to use applications for fixed, portable, or mobile non-line-of-sight services. With this technology WiMax is able to cover an entire city, not just a coffee shop or office building.

As mentioned, network security is important. In order for WiMax to be an accepted wireless service it must meet or exceed the standards already in place. This is especially important for WiMax as it can cover such large areas. Any flaws in the security and hackers would be able to break in, or there could be interference from one computer to another.

When WiFi was implemented the protocols for network security were developed. The first step for wireless security was Wired Equivalent Privacy (WEP) [3]. As more flaws were found more protocols were developed. To improve key management and initialization WEP 2 was developed. Unfortunately, this was not the answer to all the problems wireless security was faced with. Eventually, wireless security evolved to include many different protocols and encryptions that are used in WiFi today.

The lessons learned with WiFi security paved the way to the security measures used for WiMax. WiMax uses Counter Mode with Cipher Block Chaining Message Authentication Code Protocol (CCMP) to encrypt all traffic on its network. It also uses Advanced Encryption Standard (AES) to transmit data securely. Both of these are used in WiFi today and are strong in encryption and key management. Another protocol developed during the search for network security in WiFi and now used in WiMax, was PKM-EAP (Extensible Authentication Protocol). This protocol is used for end-to-end authentication. WiMax has benefited from the lessons learned with $\mathrm{WiFi}$, but there are still threats and vulnerabilities to be dealt with.

The rest of the paper is organized as follows. WiMax Security standards are discussed in Section 2, Section 3 discusses mobile wireless WiMax Security Architecture, and Section 4 discussed threats to WiMax, Conclusions are listed in Section 5. 


\section{Standards of WiMax Security}

The Protocol Stack used for WiMax is similar to that used for WiFi. The structure is the same, but WiMax uses more sublayers. The standards for WiMax Security are also similar. These standards are discussed in the following sections.

\subsection{Data Link Layer Security}

The Data Link Layer for WiMax has three sublayers. Privacy and security is handled in the bottom layer. The MAC sublayer is next, which implements secure key exchange and encrypts traffic. The last sublayer is the Service-Specific Convergence sublayer. Figure 1 shows the Protocol Stack for WiMax. The WiMax MAC layer uses a scheduling algorithm opposed to contention access used in the WiFi MAC layer. For the initial entry into the network, the scheduling algorithm attempts only once from the Subscriber Station (SS) and then it is allocated an access slot by the Base Station (BS). This access slot cannot be used by other subscribers, while assigned to the SS. To ensure confidentiality the MAC layer uses electronic signatures to authenticate the user and the device.

\subsection{Authentication}

One of the major problems that WiFi faced when first launched was authentication. Since this posed huge security issues, a better standard for authentication was used for WiMax. The WiMax network uses a Privacy Key Management (PKM) protocol for Authentication. This dynamic system makes it harder for hackers to act as a legitimate subscriber [4]. This method of authentication gives three types of protocols, a RSA based authentication, which uses a X.509 certificate with RSA encryption. An EAP based authentication, which also has three types of protocols to choose from. These three types are AKA (Authentication and Key Agreement) for SIM based authentication, TLS for X.509 based authentication, and TTLS for MS-CHAPV2 (Microsoft-Challenge Handshake

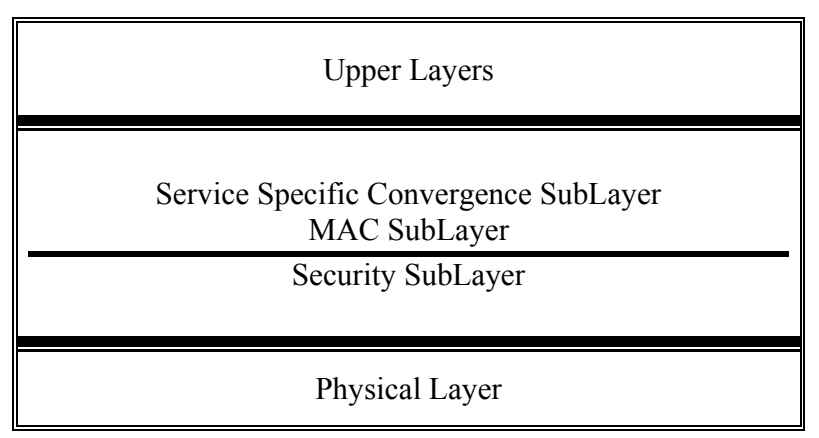

Figure 1. WiMax Protocol Stack.
Authentication Protocol). The third type of PKM protocol is RSA based authentication followed by EAP authentication [5].

\subsection{Authorization}

Authorization works hand in hand with authentication when it comes to security for WiMax. In order for a user to receive authorization, the authentication protocols must be met. Immediately following the authentication process, the SS sends an Authorization Request message to the BS [5]. In return an Authorization Reply message is sent back to the SS with the Authorization Key (AK) encrypted in the SS's public key along with the Security Association ID (SAID) of more Security Associations (SA) the SS is authorized to participate with. A lifetime key is also included with this reply. After the initial Authorization the BS periodically re-authorizes the SS.

\subsection{Encryption}

WiMax uses 3DES and AES to encrypt data transferred on the network. The Triple Data Encryption Standard (3DES) uses three different keys with a length of 56-bit each. The use of three keys causes for a slower performance in some software. The slow performance and limit on the length of keys is slowly making 3DES obsolete. The main tool for encryption that is used by WiMax is the Advanced Encryption Standard (AES) [6]. AES provides support for 128-bit, 192-bit, or 256-bit encryption keys [4]. AES was built from CCMP and has become a popular algorithm. AES is faster than 3DES, easy to implement and uses very little memory. However, it does require dedicated processors on board the BS, and may not be used by all end-user terminals. Therefore, 3DES remains a vital encryption tool on the WiMax network.

\subsection{Availability}

WiMax uses Radio Frequency (RF) Spectrum and could function on any frequency below $66 \mathrm{GHz}$ [2]. The highest frequency available in the USA is $2.5 \mathrm{GHz}$. One of the drawbacks for using RF Spectrum is that the higher the frequency the range of a BS decreases a few hundred meters. Analog TV bands may be available for WiMax use once the rollout of digital TV is complete in February of 2009.

\section{Mobile Wireless WiMax Security Architecture}

The WiMax Security Architecture is flexible to allow Base Stations of different sizes and Subscriber Stations of different functionality. It also follows the standard end-to-end architecture for the Network Reference 
Model (NRM). The network is divided into two main parts, Access Service Network (ASN) and the Connectivity Service Network (CSN). The ASN control and monitors the traffic between the Base Stations and the ASN Gateways. It also maintains the authentication and the key distributions. There are three ASN profiles, A, B, and C. Profiles A and C implements Radio Resource Management (RRM) and Handover functions, using a centralized ASN Gateway. These functions are used in the BS. Profile B embeds the key inside the BS. This eliminates the need for a centralized ASN Gateway. CSN controls the ASNs and end users with services such as AAA, Home Agent Functions, and DHCP Server. CSN also connects to operator's networks and enables inter-operator and inter-technology roaming.

\section{Threats to WiMax}

Many of the security threats found in WiMax have been addressed. These are issues that were found with the deployment of WiFi. This gives WiMax an advantage. If all, or at least most of the security issues can be addressed before WiMax's mainstream deployment, it will make it a more accepted network than WiFi. This section of the paper will discuss the known security threats in WiMax.

These include:

- Rogue base stations

- DoS attacks

- Man-in-the-middle attacks

- Network manipulation with spoofed management frames

- Threats in the physical layer

\subsection{Rogue Base Stations}

A Rogue Base Station is defined as an attacker station that imitates a legitimate base station [7]. This kind of attack results in disruptions in service and allows hackers to confuse subscribers. This is more difficult, but not impossible to do in the WiMax network. WiMax uses time division multiple access, therefore the rogue base station must transmit with a stronger strength at the same time the legitimate station transmits. The rogue base station captures the legitimate base station's identity and uses it to authenticate with the subscriber station. The authentication protocols used in WiMax help mitigate this threat. WiMax uses the EAP Protocol as its main protocol for authentication. This protocol forces mutual authentication, therefore the subscriber station would send an authentication message to the rogue base station. This does not completely alleviate the threat of rogue base stations, but it does make it more difficult.

\subsection{DoS Attacks}

Denial of Service (DoS) attacks is defined as an attempt to make a computer resource unavailable to its intended users [8]. Hackers usually use this type of attack on web servers for banks, credit card payment gateways or DNS root servers. A DoS attack uses the IP address to flood the user's network and obstruct communication between the intended user and the victim. This type of attack is not preventable; however steps can be taken to quickly resolve the attack. Some firewalls have built-in protection from DoS attacks, that monitor the amounts of packets received and the time frame they were received. It has been proposed that a Shared Authentication Information (SAI) protocol could be used to offer a defense mechanism against DoS attacks, without incurring overhead at the ASN gateway and base station. This proposal uses the unused upper 64-bit of the 128-bit Cipher Based Message Authentication Code (CMAC) to calculate a CMAC key [9]. This proposal could be the answer to prevention of DoS threats.

\subsection{Man-in-the-Middle Attacks}

Man-in-the-Middle attacks are forms of eavesdropping. The hacker establishes separate connections between two victims and relays the messages between them [10]. The hacker intercepts the public key from one of the victims and sends his or her own public key to the intended victim. When that victim responds the hacker then has that public key. The use of the RF Spectrum in WiMax allows for vulnerabilities to the man-in-the-middle attack. However, WiMax uses a three-way handshake scheme that supports re-authentication mechanisms for fast handovers to prevent man-in-the-middle attacks [11]. If the base station is constantly changed the public key changes making it almost impossible for hackers to eavesdrop using public keys.

\subsection{Network Manipulation with Spoofed Management Frames}

The management frames in WiMax are similar to WiFi's. When WiFi was first deployed vulnerabilities were found in the management frames that allowed DoS attacks by disrupting the wireless session between two nodes. WiMax has cryptographic protections from spoofed identities, but that does not mean it is safe. Replay DoS attacks still remain a threat to WiMax, due to the lack of any mechanisms to specifically detect and discard repeated packets [12].

\subsection{Threats in the Physical Layer}

Blocking and rushing are the major threats located in the 
physical layer. Blocking or jamming activates a strong frequency to lower the capacity of the channel creating a DoS to all stations. This threat is detectable with a radio analyzer device. This device does not prevent this threat, but it alerts the end-user so that steps can be taken to immediately recover. Rushing or scrambling is another type of jamming, but it only activates for short periods of time and only affects certain frames. Jamming can be prevented using an increased signal or using frequency hopping. Control or Management messages are not in danger of rushing or blocking. Scrambling the uplink slots is too difficult for hackers [13].

\section{Conclusions}

WiMax security has been discussed in this paper. The lessons learned with WiFi's deployment have given WiMax an advantage in providing a safer wireless network. The precautions taken with WiMax were not done for WiFi. These security measures were not taken at WiFi's launch because the threats were unknown. Now that the threats are known and understood, they have been addressed prior to WiMax's deployment. However, this does not mean that WiMax is flawless. There may be new threats that are unknown and will not be addressed until WiMax is launched. WiMax does have much potential. WiMax would allow customers a completely wire free network connection in their homes or businesses. WiMax would also provide better services for mobile devices. There are also some speculations for WiMax to be used in gaming consoles. While security is an important subject and was a main cause of delay in the first stages of development for WiMax, it has now been faced with technological down falls. Sprint Nextel is one of the main companies looking to bring WiMax to the US. The main cause for delay has been the Sprint Nextel backhaul links from the backbone to the towers. It has been unable to support the promised $4 \mathrm{Mbps}$. Until recently there has been little hope that a resolution to this problem would surface. In July 2008, Sprint Nextel announced that a solution has been found. They will be teaming up with DragonWave, a Canadian company, to provide the backhaul links. This will allow all data passing from the backbone to the end user to travel over the air, and the towers will need only power links. With this new development a testing launch of WiMax to Portland, Oregon is ready now, and preparations for a launch in 2009 to
LasVegas, Atlanta, and Grand Rapids. If these tests go well we could see a full commercial launch by 2010 . However, Clearwire has successfully deployed WiMax to several cities in the US and has been the main WiMax service provider for the last few years. With the formation of the Open Patent Alliance (OPA), which includes Clearwire, Sprint, Alcatel-Lucent, Cisco, Intel Corporation, and Samsung Electronics, WiMax could be global in the next five years.

\section{References}

[1] A. S. Tanenbaum, "Computer Networks," 4th Edition, Prentice Hall, Inc., New Jersey, 2006.

[2] http://en.wikipedia.org/wiki/WiMax

[3] S. P. Ahuja and P. K. Potti, "Evolution of Wireless LAN Security," International Conference on Parallel and Distributed Processing Techniques and Applications, Las Vegas, 24-17 July 2008.

[4] T. Sanders, "Premium Five Essential Elements of WiMax Security," WiMax.com, November 2007.

[5] http://myhsc.pbwiki.com/wimax::aaa

[6] P. Korsenlowski, "Staying Safe in a WiMax World," TechNewsWorld.com, 27 February 2007.

[7] M. Barbeau, J. Hall and E. Kranakis, "Detecting Impersonation Attacks in Future Wireless and Mobile Networks," Secure Mobile Ad-hoc Networks and Sensors, Ottawa, 2006, pp. 80-95.

[8] M. McDowell, "Understanding Denial of Service Attacks," National Cyber Alert System, 1 August 2007.

[9] K. Youngwook, L. Hyoung-Kyu and B. Saewoong, "Shared Authentication Information for Preventing DDoS Attacks in Mobile WiMax Networks," Proceedings of the 5th Consumer Communications and Networking Conference, Las Vegas, 10-12 January 2008.

[10] N. Beacham, "Man in the Middle (MITM) Attacks," Technology and More, 28 June 2008.

[11] J. M. Hartley, "WiFi and WiMax Protocols of Security," December 2008. http://softwarecommunity.intel.com/articles/eng/3708.htm

[12] R. Millman, "Security Experts See Vulnerablilites in WiMax," WiMax.com, 17 October 2006.

[13] M. Barbeau, "Threats: Threats to WiMax," http://www. freewimaxinfo.com/physical-layer.html 